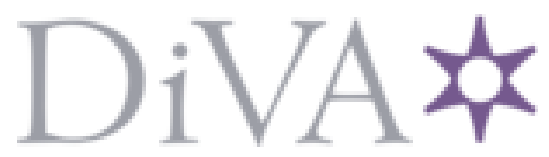

http://www.diva-portal.org

This is the published version of a paper presented at The 2013 IEEE International Conference on Internet of Things, Beijing, China, 20-23 August 2013.

Citation for the original published paper:

Glaropoulos, I., Mangold, S., Vukadinovic, V. (2013)

Enhanced IEEE 802.11 Power Saving for Multi-Hop Toy-to-Toy Communication.

In: IEEE Computer Society

N.B. When citing this work, cite the original published paper.

Permanent link to this version:

http://urn.kb.se/resolve?urn=urn:nbn:se:kth:diva-12857 1 


\title{
Enhanced IEEE 802.11 Power Saving for Multi-Hop Toy-to-Toy Communication
}

\author{
Ioannis Glaropoulos, Stefan Mangold, Vladimir Vukadinovic \\ Disney Research \\ 8092 Zurich, Switzerland
}

\begin{abstract}
In the future Internet of Things (IoT), batterypowered devices equipped with short range radios may need to communicate with each other over multi-hop links. This may significantly increase their energy consumption. Whereas most research on IoT assumes that the devices use energy-efficient IEEE 802.15.4 wireless transceivers, we focus on IEEE 802.11 because of its wide penetration in consumer electronics such as toys. We extend the IEEE 802.11 power saving mode (PSM), which allows the devices to enter the low-power doze state, with a traffic announcement scheme that facilitates multi-hop communication. The scheme propagates traffic announcements along multi-hop paths to ensure that all intermediate nodes remain awake to forward the pending data frames with minimum latency. Simulation results show that the proposed Multi-Hop PSM (MH-PSM) improves both end-to-end delay and doze time compared to the standard PSM. MH-PSM is practical and software-implementable since it does not require changes to the parts of the IEEE 802.11 medium access control that are typically implemented in hardware.
\end{abstract}

\section{INTRODUCTION}

Many objects, such as consumer electronics and toys, are becoming equipped with sensors and wireless communication capabilities. They will connect to each other locally using ad hoc networks and globally using IP infrastructure to create the Internet of Things (IoT). Ad hoc networks enables wireless devices to exchange data with one another when a fixed network infrastructure (access points, cellular base stations) is not available. In ad hoc networks, communication between devices that are out of each other's transmission range is established over multi-hop routes. Hence, each device is not only receiving and transmitting its own data, but it also serves as a multi-hop relay for other devices. This increases the energy consumption and decreases the battery lifetime of the devices. Therefore, one of the major challenges for ad hoc networking of battery-powered devices is the energyefficiency of radio communication. Most of the on-going research on IoT assumes that the devices are equipped with low-power IEEE 802.15.4 (Zigbee) transceivers. However, for consumer electronics, such as radio-enabled toys, the wide penetration of IEEE 802.11 (Wi-Fi) dictates the choice of the wireless technology. The energy consumption of 802.11 is high compared to 802.15.4 and other low-power radios. To alleviate this problem, the 802.11 standard [1] already specifies Power-Saving Mode (PSM) that allows idle 802.11 stations to transition to a low-power doze state.

An 802.11 station in PSM mode wakes up periodically from the doze state, listens for traffic announcements coming

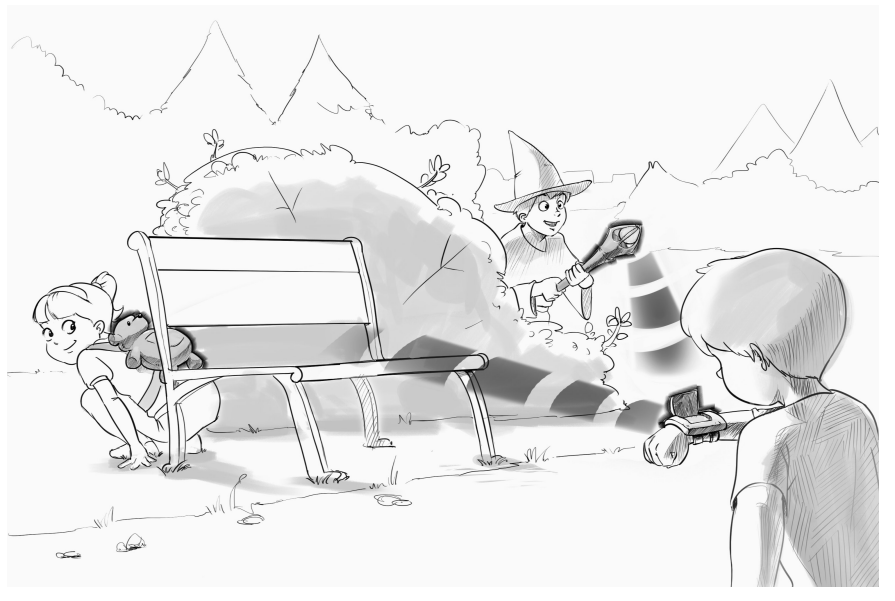

Fig. 1. Application scenario: Multi-hop communication between toys in an outdoor game. Devices communicate directly with each other, without network infrastructure.

from other stations that have data packets destined for it, and announces its own data packets destined for other stations. If a STA does not receive any traffic announcements and it does not have buffered packets that can be transmitted, it returns to the doze state. The standard [1] specifies the details of PSM mechanism for both infrastructure/BSS mode (Basic Service Set with an access point) and ad hoc/IBSS mode (Independent Basic Service Set without an access point). In the ad hoc mode, especially in muti-hop networks, the PSM is known to perform poorly, causing undesirable energy consumption and/or long packet delivery delays [2, 3, 4]. The reason is because PSM has been originally designed for single-hop communication in the infrastructure mode (from the access point to a station and vice versa). When a data frame is forwarded over multiple hops, standard 802.11 PSM may significantly increase its delivery delay because only the next-hop station is notified about the pending frame via traffic announcements - the stations on subsequent hops may remain in the doze state. Therefore, in each beacon interval the frame is forwarded over a single hop and has to be buffered before being forwarded further.

In this paper, we propose a mechanism that wakes up downstream stations so that data frames can be forwarded over multiple hops in a single beacon interval. This is achieved by instructing each station along the path to send a traffic announcement to its downstream neighbor. The proposed mechanism significantly reduces the end-to-end latency, es- 


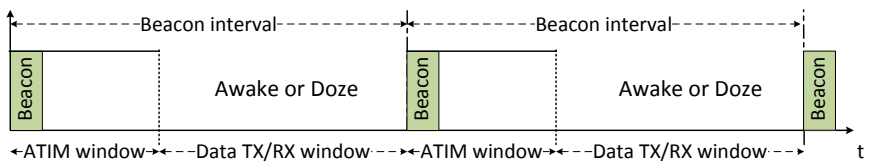

Fig. 2. 802.11 PSM divides each beacon interval into an ATIM window and a data TX/RX window.

pecially for bursty traffic where intermediate stations may move to the doze state between two consecutive traffic bursts. The mechanism enhances the standard PSM to what we call multi-hop PSM (MH-PSM). MH-PSM does not prevents stations to inter-operate with those that run standard PSM it does not alter the state machine, frame formats, and other important elements of the protocol. MH-PSM is also softwareimplementable - it does not require modifications to the parts of the 802.11 MAC protocol that are usually implemented in hardware, such as the CSMA/CA and handling of control frames (RTS, CTS, ACK).

The rest of the paper is organized as follows: Section II summarizes the standard 802.11 PSM. In Section III we describe MH-PSM and discuss practical implementation issues. The performance of the mechanism is evaluated in Section IV using simulations. In Section V, we provide an overview of related work. Finally, Section VI concludes the paper.

\section{POWER-SAVING MODE FOR 802.11 AD HOC NETWORKS}

In the standard 802.11 PSM for ad hoc/IBSS networks, time is divided into periods called beacon intervals. Each station wakes up at the beginning of each beacon interval and starts a back-off procedure in an attempt to transmit a beacon. If a station receives a beacon from another station before its back-off timer expires, it cancels the pending beacon transmission. The Timing Synchronization Function (TSF) uses the time-stamped beacons to synchronize clocks among stations to ensure that all stations wake up at the same time. Following the beacon exchange, each station stays awake for an ATIM window interval, as shown in Fig. 2. During the ATIM window, stations announce pending data frames to their neighbors using unicast announcement traffic indication messages (ATIMs). ATIMs are sent using 802.11 distributed coordination function (DCF), which implements CSMA/CA channel access procedure. A station that receives an ATIM should respond with an ACK. Successful exchange of ATIMACK packets between two stations implies that they can now exchange any pending data frames and thus both should stay awake until the next beacon interval. Stations that do not send nor receive any ATIM frame during an ATIM window will move to the doze state for the rest of the beacon interval. After the end of ATIM window, all stations that remain awake will follow the normal DCF procedure to transmit and receive data frames.

The described PSM protocol has many drawbacks. For example, when a station successfully transmits or receives an ATIM frame during an ATIM window, it must stay awake during the entire beacon interval. At low loads, this approach results in a much higher energy consumption than necessary. Another shortcoming is that all stations in an IBSS must use the same fixed ATIM window size, which is set at the time when the IBSS is created, as well as identical beacon intervals. Since the ATIM window size critically affects the throughput and energy consumption, the fixed ATIM window does not perform well in all situations, as shown in [5]. Some of these drawbacks have been addressed in previous works, which are mentioned in the related work section. This paper, however, addresses the problem of end-of-end delay on multi-hop paths, which is described in the following.

Consider a scenario where station A needs to send a single frame/message to station D using intermediate stations B and $\mathrm{C}$ as relays (Fig. 3). In the first beacon interval, station A announces the data frame to station B using an ATIM frame. Station B acknowledges the ATIM an remains awake so that it can receive the data following the ATIM window. Station $\mathrm{C}$ has not received any ATIMs and, therefore, it enters the doze state. Since station B is not able to forward the frame to $\mathrm{C}$ in the current beacon interval, it has to wait for the start of the next beacon interval to send an ATIM to station C. Following a successful ATIM-ACK exchange, the frame is forwarded to C. Station D will receive the frame in the third beacon interval. The resulting end-to-end delay may considerably affect network applications with strict latency constraints. Therefore, enabling PSM in multi-hop ad hoc networks must be combined with effective mechanisms for mitigating its effect on the resulting packet delays.

\section{ENHANCED 802.11 PSM FOR MUlTi-HoP COMMUNICATION}

In the above described scenario, the data frame sent by $\mathrm{A}$ must be buffered at B before it is relayed to $\mathrm{C}$ in the following beacon interval. This could have been avoided if there was a way for B to, upon receiving the ATIM from A, send an early ATIM to $C$ and $D$ to inform them about the pending data frame at A. This is what our low-latency multi-hop PSM (MH-PSM) aims to achieve.

Before introducing MH-PSM, we describe the format of ATIM frames. An ATIM frame includes a MAC header, whose structure shown in Fig. 4 is common to all management frames. The header includes three address fields: Address 1 contains the MAC address of the ATIM receiver. Address 2 contains the MAC address of the ATIM sender. Address 3 may contain different information depending on the type of the management frame and network (BSS, IBSS, or mesh). In case of an ATIM frame, Address 3 contains the BSSID (BSS identifier) of the IBSS, but this identifier is not used. The frame body of an ATIM is empty.

\section{A. Proposed Extension: Multi-Hop PSM (MH-PSM)}

We propose that, in order to inform all stations along the path to D about the pending data frame, the station A writes the MAC address of D into the Address 3 field of the ATIM frame that are sent to $\mathrm{B}$. The methods that $\mathrm{A}$ can use to resolve the MAC address of D from its IP address are discussed later 


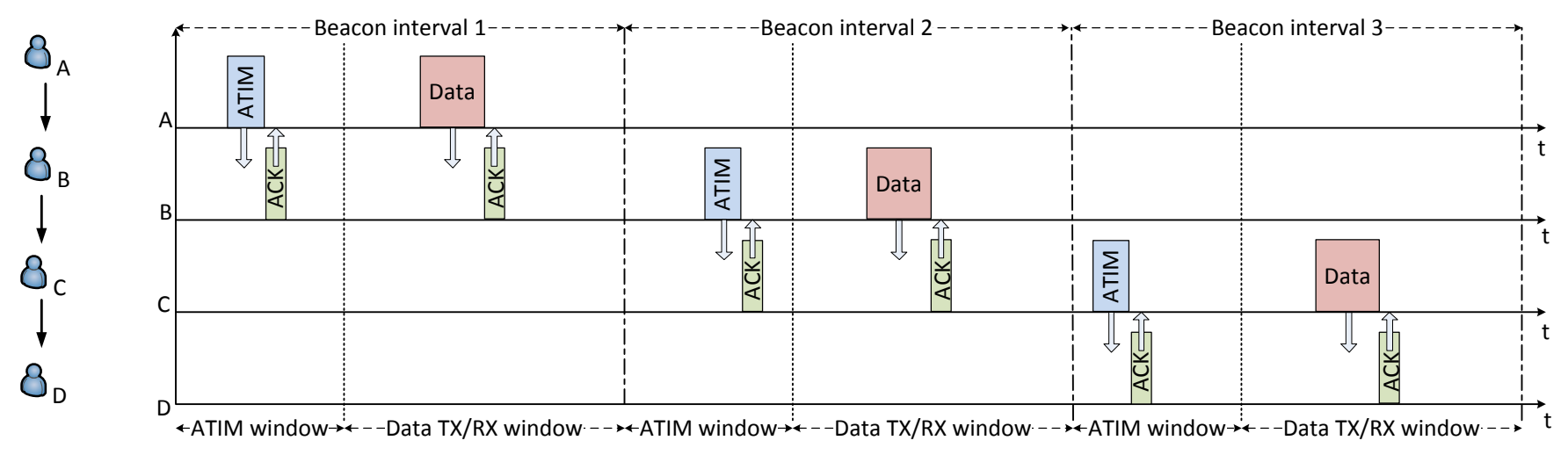

Fig. 3. Multi-hop forwarding in standard 802.11 PSM may cause a delay of several beacon intervals.

in this Section. Upon receiving the ATIM, B inspects the Address 3 field to derive the final destination of the data frame announced by that ATIM. It retrieves the MAC address of D from the Address 3 field, resolves it to the IP address of D, and consults the routing table to find out that $\mathrm{C}$ is the next hop on the path to D. Then B creates an ATIM frame for C with the MAC address of D inside the Address 3 field. When $C$ receives the ATIM from B, it uses the same procedure to create an ATIM for D. In this way, a "wave" of ATIMs is created along the path to wake up all relays and the destination of the data frame. Following the end of the ATIM window, the data frame is forwarded end-to-end since all stations on the path are in the awake state. The procedure is illustrated in Fig. 5. The ATIM wave may not reach the end destination: it may terminate at the end of the ATIM window or upon reaching a station that cannot resolve the MAC address of the destination. In that case, the data frame will be forwarded in the current beacon interval as far as the station that has received the last ATIM in the sequence. Nevertheless, MH-PSM may significantly decrease the end-to-end delay because the probability that data frames are forwarded over multiple hops in a single beacon interval is higher than with the standard PSM.

\section{B. Address 3 Resolution}

The sending station A needs to store the MAC address of the destination D into the Address 3 field of ATIMs sent to B. Therefore, A needs to resolve the MAC address of D from its IP address. Since the paper is targeting Internet of Things (IoT) and smart toy communication scenarios, we assume that IPv6 is used. IPv6 protocol suite uses Neighbor Discovery (ND) protocol [6] for address resolution, nexthop determination, and duplicate address detection. Address resolution enables stations to determine MAC addresses of

\begin{tabular}{|c|c|c|c|c|c|c|}
\hline $\begin{array}{c}\text { Frame } \\
\text { Control }\end{array}$ & Duration & Address 1 & Address 2 & Address 3 & $\begin{array}{c}\text { Sequence } \\
\text { Control }\end{array}$ & FCS \\
\hline
\end{tabular}

Fig. 4. Structure of the ATIM frame. The Address 3 field can be used for the MAC address of the end destination. their neighbors given only their IP addresses. The neighbor solicitation messages, which are used for address resolution, are sent via multicast. The ND protocol is not designed with multi-hop ad hoc networks in mind. A node in such network is able to broadcast to other nodes within its radio range, but the communication is non-transitive. Therefore, a wireless ad hoc network is a non-broadcast multi-access (NBMA) structure with generally no network-wide multicast capabilities. The network solicitation messages are not forwarded in an IBSS. Hence, station A is only able to resolve MAC addresses of its immediate neighbors, but not of $\mathrm{D}$, which is multiple hops away. There are several proposals to extend the capabilities of the ND protocol to multi-hop ad hoc networks [7] and 6LoWPAN networks in particular [8]. These proposals include mechanisms for multi-hop duplicate address detection (DAD), which allows a station to check the uniqueness of an IP address in an $n$-hop neighborhood. The multi-hop DAD can also be used for multi-hop address resolution: station A may initiate multi-hop DAD for the IP address of D. Upon receiving a DAD request, $\mathrm{D}$ will respond with a DAD confirmation message that contains its MAC address. It this way, A can resolve the MAC address of D based on its IP address. Note that each station maintains a cache of resolved addresses, which reduces the need for network-wide multi-hop address resolution.

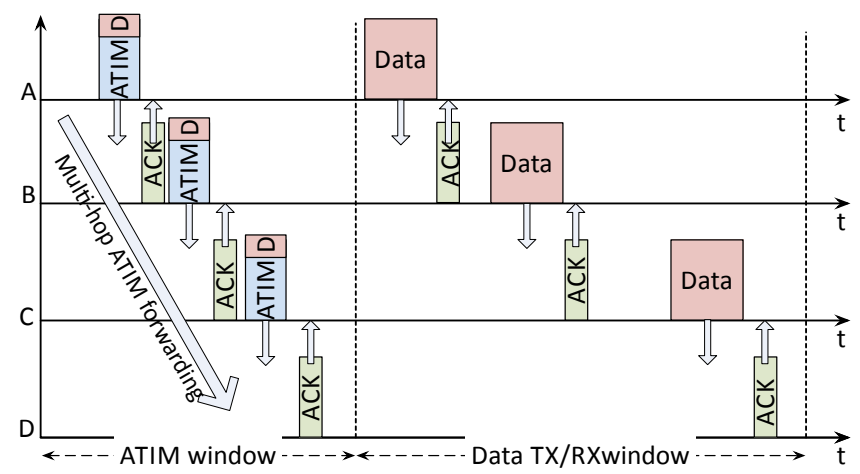

Fig. 5. The proposed multi-hop forwarding mechanism allows data frames to be forwarded end-to-end in a single beacon interval. 
TABLE I

DEFAULT SIMULATION PARAMETERS

\begin{tabular}{r|l} 
Parameter & Value \\
\hline Grid size & $7 \times 7$ stations \\
Grid spacing & $50 \mathrm{~m}$ \\
Channel model & unit disk \\
IEEE 802.11 PHY mode & $11 \mathrm{Mb} / \mathrm{s}(802.11 \mathrm{~b})$ \\
Short / long retry limit & $4 / 7$ (Threshold: 500 B) \\
MAC buffer size & 100 frames \\
Beacon interval & $50 \mathrm{~ms}$ \\
ATIM window & $10 \mathrm{~ms}$ \\
Traffic model & Poisson $(\lambda)$ \\
Data frame size & Uniform $[50,1500]$
\end{tabular}

\section{Backward-Compatibility and Software Implementation}

Backward-compatibility with the standard PSM is ensured since MH-PSM does not violate neither frame formats nor protocol operations. Stations that implement standard PSM will not check the Address 3 field of received ATIMs and, therefore, the wave of ATIMs will terminate at such stations. This diminishes some of the delay improvements, but otherwise does not prevent or impair communication. $\mathrm{MH}-$ PSM is also software-implementable: Parsing and creation of ATIM frames are not time-critical operations that have to be implemented in hardware. This enables driver-level implementation of MH-PSM without modifications to the lowlevel MAC operations. We are currently implementing $\mathrm{MH}-$ PSM in an Atheros AR9170 driver.

\section{PERformance Evaluation}

We compared the performance of standard PSM and $\mathrm{MH}-$ PSM using simulations. The performance is measured in terms of end-to-end delay, doze time ratio, ATIM overhead, and packet delivery ratio, as defined below:

End-to-End Delay is the average time required to forward a data frame from a source to its destination over multiple hops. It is averaged over all successfully delivered data frames.

Doze Time Ratio is the percentage of beacon intervals in which a station enters doze state, which closely correlates with the energy consumption. It is averaged over all stations that participate in traffic forwarding.

ATIM Overhead is the average number of ATIM frames sent per one successfully delivered data frame. The relative ATIM overhead of MH-PSM is the ratio of ATIM overheads obtained with MH-PSM and standard PSM.

Packet Delivery Ratio (PDR) is the percentage of data frames that are successfully delivered to the end destination. A station may drop a data frame if it exceeds the maximum number of retransmissions.

The simulation setup and the results are described in the following:

\section{A. Simulation Setup}

We implement and tested MH-PSM in Jemula802 [9], which is a Java-based 802.11 protocol simulator developed in our group. We consider a regular $7 \times 7$ grid of static 802.11 stations. Adjacent nodes are $50 \mathrm{~m}$ apart from each other. We

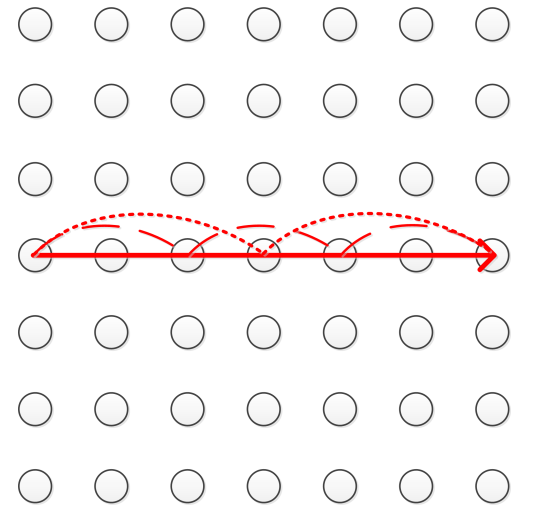

Fig. 6. Simulated network topology with a single flow. The transmission range is set to $50 \mathrm{~m}, 100 \mathrm{~m}$, and $150 \mathrm{~m}$ to produce paths with 2,3 , and 6 hops, respectively.

assume a simple unit disk radio propagation model. We varied the radio range from $50 \mathrm{~m}$ to $150 \mathrm{~m}$ to influence the number of hops between source-destination pairs. The beacon interval and ATIM window size are $50 \mathrm{~ms}$ and $10 \mathrm{~ms}$, respectively, unless stated otherwise. The data traffic is Poisson (exponential interarrival times) with uniformly distributed frame sizes. The number of active flows and mean frame interarrival time are varied to control the load in the network. The traffic is routed over shortest paths; the routing is static. We ensured that the simulation duration is sufficient to make the variations in time-moving averages insignificant. The default simulation parameters are summarized in Table I.

\section{B. Simulation Results}

Consider first the simple single-flow scenario shown in Fig. 6, where the station in the first column of the grid is sending data frames to the station in the last column over multiple hops. Note that non-forwarding nodes in the grid affect the performance of forwarding nodes: The 802.11 standard mandates that a station that transmits a beacon should remain awake for the rest of the beacon interval. As the number of its neighbors decreases, the probability that a station transmits a beacon before it receives one increases. The radio transmission range is set to $50 \mathrm{~m}, 100 \mathrm{~m}$, and $150 \mathrm{~m}$ in different simulation runs, which produces paths with 2,3 , and 6 hops, respectively. On average, the sender is generating $\lambda=10$ frames per second ( 0.5 frames per beacon interval).

The results for the average end-to-end frame delay are shown in Fig. 7 (left). As expected, the delay increases with the number of hops. For the standard PSM it takes almost $N$ beacon intervals to forward a frame over $N$ hops. It may happen that a frame is forwarded over multiple hops in a single beacon interval: if its next-hop neighbor is awake, a station may immediately forward the frame to it, without waiting for the next ATIM window to send a traffic announcement. In a lightly loaded network, however, it is likely that the next-hop station is in the doze state, and therefore, the data frame has to be announced with an ATIM in the next beacon interval. The results show that the delay is significantly 

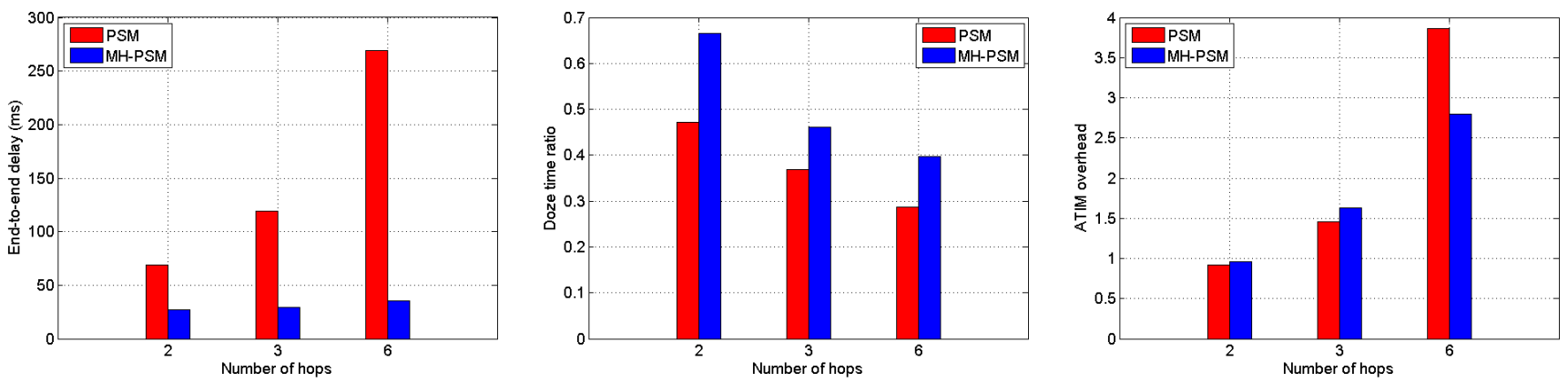

Fig. 7. End-to-end delay, doze time ratio, and ATIM overhead for different numbers of hops.

shorter for MH-PSM. Although it slightly increases with the number of hops (due to processing in intermediate stations and increasing probability of collisions/retransmissions caused by hidden stations) the average delay is well below $50 \mathrm{~ms}$, which is the duration of the beacon interval. As the number of hops increases from two to six, the percentage of frames that are forwarded end-to-end within a single beacon interval deceases from $88 \%$ to $86 \%$, but still remains exceptionally high compared to standard PSM (28\% and 0\%).

The average doze time ratio is shown in in Fig. 7 (middle). The results show that MH-PSM significantly increases the energy efficiency by allowing the stations to move to the doze state more often than the standard PSM. The reason for this is that MH-PSM prevents excessive buffering of frames in intermediate stations, which effectively decreases the traffic load and the probability of collisions/retransmissions. The sixhop packet delivery ratio for MH-PSM is $99.4 \%$ versus $91.5 \%$ for the standard PSM. The results presented so far show that MH-PSM provides both shorter delay and lower energy consumption, which is a major improvement over the standard PSM whose parametric adjustments/optimizations may only trade shorter delay for higher energy consumption and vice versa.

In Fig. 7 (right), we show the ATIM overhead for both PSM schemes. While the overhead for MH-PSM is slightly higher for paths with few hops (i.e. two or three), the opposite is true for the six-hop path where it results in $25 \%$ overhead reduction compared to the standard PSM. To understand the reasons for the trend reversal, consider a five-hop path from station A to station $\mathrm{E}$ via $\mathrm{B}, \mathrm{C}$, and $\mathrm{D}$, as shown in Fig. 8. Assume that one frame is buffered at station $\mathrm{A}$ and one at station $\mathrm{C}$. In the best-case scenario, it will take four beacon intervals and six ATIMs to deliver both frames to the destination with the standard PSM. With MH-PSM however, it will only one beacon interval and four ATIMs to achieve the same because it creates a wave of ATIMs that flushes all buffered frames to the destination, as shown in Fig. 9.

There are however scenarios where the ATIM overhead of MH-PSM is higher than that of the standard PSM, even for paths with many hops. In the standard PSM, a station sends a single ATIM to its neighbor to announce all data frames that it intends to forward to this neighbor, regardless of their end destinations. In MH-PSM, the station may send multiple ATIMs with different Address 3 fields to the neighbor if the pending data frames have different end destinations. For example, consider two flows whose eight-hop paths contain a common subset or relays, as shown in Fig. 10. In MH-PSM, the common relays may need to forward two ATIMs with different Address 3 fields to their next-hop neighbors in the same ATIM window. This is not the case in standard PSM, where only one ATIM is sent. The results in Fig. 11 show that the ATIM overhead of MH-PSM is almost $60 \%$ higher in this scenario. However, MH-PSM outperforms standard PSM in all other respects: the end-to-end delay is close to tenfold shorter, the doze time ratio is slightly higher, and the packet delivery ratio is significantly improved. Therefore, the relative ATIM overhead of MH-PSM had no bearing to the key performance metrics.

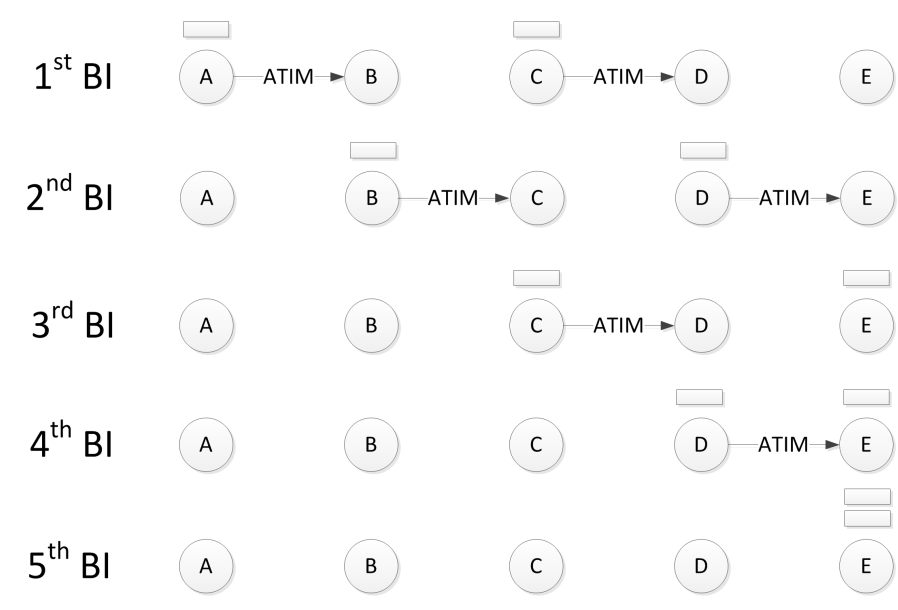

Fig. 8. Standard PSM requires 4 BIs and 6 ATIMs to deliver the frames buffered at $\mathrm{A}$ and $\mathrm{C}$.

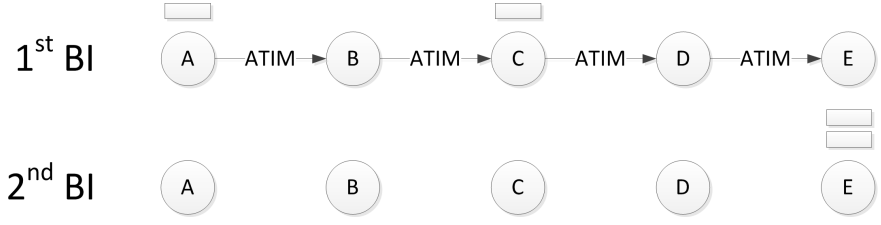

Fig. 9. MH-PSM requires only $1 \mathrm{BI}$ and 5 ATIMs to deliver the frames buffered at $\mathrm{A}$ and $\mathrm{C}$. 


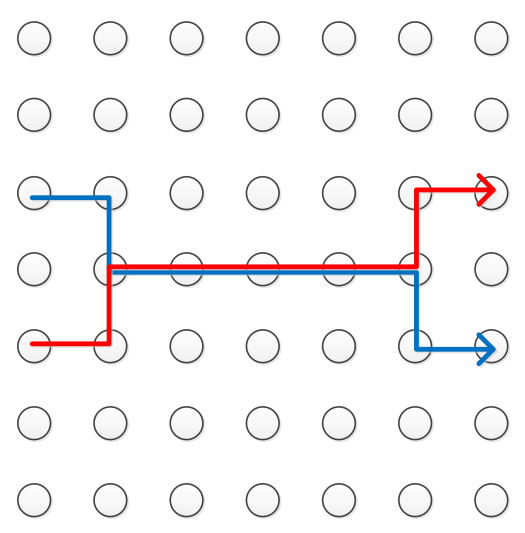

Fig. 10. An example of two flows whose paths partially overlap.

TABLE II

PERFORMANCE OF STANDARD PSM AND MH-PSM FOR DIFFERENT BEACON INTERVALS. THE TRANSMISSION RANGE IS $50 \mathrm{M}$ - FRAMES ARE FORWARDED OVER SIX HOPS.

\begin{tabular}{|c|c|c|c|c|c|c|}
\hline \multirow{2}{*}{ Bcn. Int. } & \multicolumn{2}{|c|}{ Delay (ms) } & \multicolumn{2}{c|}{ Doze time (\%) } & \multicolumn{2}{c|}{ PDR (\%) } \\
\cline { 2 - 7 } & PSM & MH-PSM & PSM & MH-PSM & PSM & MH-PSM \\
\hline $50 \mathrm{~ms}$ & 269.08 & 35.44 & 0.29 & 0.40 & 91.54 & 99.36 \\
\hline $100 \mathrm{~ms}$ & 514.84 & 47.15 & 0.15 & 0.26 & 85.43 & 99.17 \\
\hline
\end{tabular}

We next investigate the impact of beacon interval on the performance of standard PSM and MH-PSM. The results presented so far assume a beacon interval of $50 \mathrm{~ms}$. We increased the beacon interval to $100 \mathrm{~ms}$ and repeated the simulations for the basic scenario shown in Fig. 6 with the transmission range of $50 \mathrm{~m}$ (i.e. six hops). The average frame interarrival time is $100 \mathrm{~ms}$. The results are summarized in Table II. As expected, the frame delay for PSM doubles because the time that frames stay buffered in the intermediate nodes is proportional to the beacon interval. The delay for MH-PSM also increases, but the increase is comparably modest. The increase is due to the fact that MH-PSM does not guarantee that all frames will be delivered end-to-end in a single beacon interval. Some of the frames have to be buffered along the path as in the case of standard PSM. Another observation is that with the standard PSM packet delivery ratio decreases significantly for the longer beacon interval (from $91.5 \%$ to only $85.4 \%$ ),

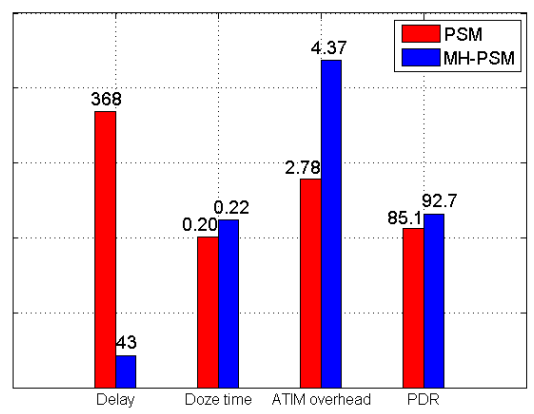

Fig. 11. Performance of standard PSM and MH-PSM for the scenario with two flows whose paths partially overlap. while with MH-PSM it decreases only slightly (from $99.4 \%$ to $99.2 \%$ ). With the standard PSM, the number of buffered frames along the path increases with the duration of the beacon interval, which effectively increases the traffic load in the network and the probability of collisions. With MH-PSM, most frames are delivered end-to-end without buffering in the intermediate nodes.

In our final set of simulations, we consider multiple intersecting flows in the grid. The scenarios with 2, 4, and 8 flows shown in Fig. 12 complement the single-flow scenario in Fig. 6. The transmission range is set to $50 \mathrm{~m}$ and, therefore, frames are forwarded over six hops. The results in Table III show that the performance deteriorates with the number of flows. Transmissions of intersecting nodes are especially prone to collisions because they are surrounded by four active/forwarding stations that do not hear each others transmissions ("hidden stations"). The impact of collisions on the performances of the standard PSM and MH-PSM is somewhat different: While the frame delay for the standard PSM remains unaffected by the number of flows, the delay for MH-PSM increases considerably (yet still remains relatively low). The reason is that collisions in intersecting nodes may disrupt the cut-through forwarding of data frames in MH-PSM. In the single-flow scenario, $88 \%$ of frames are forwarded end-to-end in a single beacon interval. In the eight-flow scenario, this percentage drops to $79 \%$. The additional hold-up in intersecting nodes does not affect the frame delay in the standard PSM so prominently because most frames are anyway forwarded only one hop per beacon interval.

\section{Ongoing Work and Open Issues}

We are currently implementing the proposed MH-PSM on a hardware platform shown in Fig. 13. The platform consists of an Arduino Due board with ARM Cortex-M3 microprocessor and $9 \mathrm{~KB}$ of SRAM [10] and an 802.11n transceiver based on Atheros AR9170 chipset [11]. The platform runs Contiki operating system [12]. MH-PSM will be implemented as a part of a Contiki Wi-Fi driver for AR9170. We are planning to validate the simulation results on a testbed of 25 devices. We will further investigate the impact of ATIM window size and beacon interval on delay and energy consumption of $\mathrm{MH}-$ PSM. According to the 802.11 standard, the beacon interval and ATIM window are determined at the time when an IBSS is created and shall be static for the lifetime of the IBSS. A short ATIM window reduces the energy spent while listening

TABLE III

PERFORMANCE OF STANDARD PSM AND MH-PSM FOR DIFFERENT NUMBERS OF FLOWS. THE TRANSMISSION RANGE IS $50 \mathrm{M}-$ FRAMES ARE FORWARDED OVER SIX HOPS.

\begin{tabular}{|c|c|c|c|c|c|c|}
\hline \multirow{2}{*}{ Num. flows } & \multicolumn{2}{|c|}{ Delay (ms) } & \multicolumn{2}{c|}{ Doze time (\%) } & \multicolumn{2}{c|}{ PDR (\%) } \\
\cline { 2 - 7 } & PSM & MH-PSM & PSM & MH-PSM & PSM & MH-PSM \\
\hline 1 & 269.08 & 35.44 & 0.29 & 0.40 & 91.50 & 99.36 \\
\hline 2 & 269.07 & 39.78 & 0.21 & 0.37 & 83.72 & 92.44 \\
\hline 4 & 269.34 & 42.28 & 0.19 & 0.34 & 81.74 & 86.88 \\
\hline 8 & 273.37 & 46.73 & 0.17 & 0.24 & 80.01 & 85.80 \\
\hline
\end{tabular}



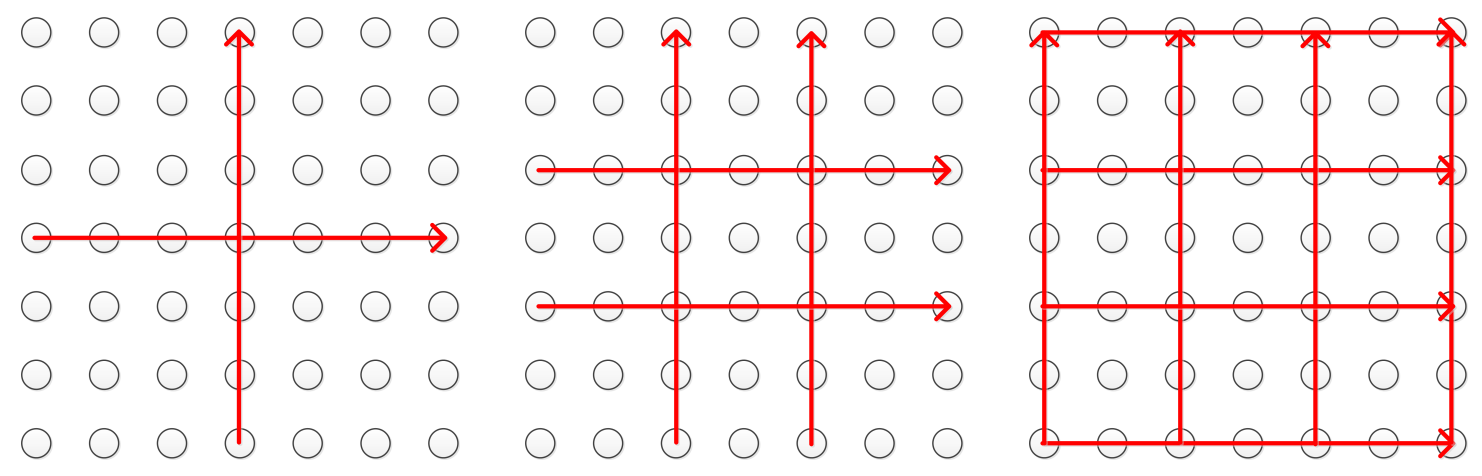

Fig. 12. Simulated network topology with 2, 4, and 8 simetric flows.

for ATIMs. However, if it is too short, it might not provide enough time to announce all pending frames, which decreases the throughput. If the ATIM window is too long, there might be not enough time to transmit all announced data frames in the post-ATIM window. The size of the ATIM window can be set based on the expected traffic load in the network lower load implies shorter ATIM window to minimize the energy consumption. MH-PSM introduces an additional tradeoff: Even at a low traffic load, a longer ATIM window might be needed in order to propagate the wave of ATIMs end-toend, which means that the remaining post-ATIM window may be too short to forward the announced data frames end-to-end. Hence, some of the downstream stations might be awaken for no reason. Therefore, in MH-PSM, the choice of the ATIM window size depends not only on the expected traffic load, but also on the expected number of hops to the destination. We are also planning to investigate the interactions of MH-PSM with upper layers (routing, transport) and their joint performance under node mobility.

\section{RELATED WORK}

The IEEE 802.11ah proposal [13] defines a low power medium access method that optimizes standard 802.11 PSM for battery-powered devices used in smart metering and machine-to-machine communication. However, the optimization focuses on BSS (infrastructure) networks where PSMenabled stations communicate with an access point.

Optimization of PSM for IBSS (ad-hoc) networks has attracted considerable attention in the research community. A number of approaches focuses on minimizing the duration of idle listening by introducing mechanisms for early transition to the doze state $[2,3,4]$. In [2], the explicit announcement of the number of pending frames in ATIMs is proposed in order to allow the receiving station to move to the doze state after it receives the last frame, instead of waiting for the end of the beacon interval. In [14], the authors propose a scheme where ATIMs contain information about the nature of the intended traffic, so stations can differentiate between broadcast and multi-cast traffic; in the later case they can immediately transit to doze state if they are not members of the multicast group. In various approaches, the early transition to the doze state is combined with the dynamic adjustment of the ATIM window duration, depending on the traffic conditions in the IBSS [15]. In [3] the authors propose an algorithm for a station to dynamically adjust the remaining ATIM window duration as a response to ATIM receptions in order to transit to sleep earlier in case of low network traffic.To further decrease the energy wasted for idle listening, [16] proposes a scheme where transmitting stations announce their intention of sending ATIM frames in a short time period at the beginning of the beacon interval. Stations that do not send or receive any announcements do not have to stay awake for the entire ATIM window. Considering a similar low-traffic scenario, [17] proposes a scheme where the absence of traffic is declared by transmitting a delayed beacon, so that stations can skip idle listening during the ATIM window. In $[18,19]$, the authors propose a topologyaware power-saving algorithm based on the overhearing of the ATIM frames transmitted by the neighbors. By extracting the source addresses from the received ATIM acknowledgments, a station can defer from transmitting ATIMs to stations known to remain awake after the expiration of the ATIM window. This scheme can efficiently decrease the required ATIM window size in a fully-connected IEEE 802.11 mesh network, but it is less effective in multi-hop IBSS network topologies.

Optimizations of PSM for multi-hop IBSS networks have also been proposed in several papers. For example, [4] introduces an ad-hoc clustering scheme where master nodes form a backbone that relays the multi-hop traffic between PSMenabled slave nodes and proposes a distributed algorithm for dynamical and fair selection of master nodes in an IBSS. In [20], stations increase their energy saving by waking-up at multiples of the beacon period and utilize an adaptive next-hop selection framework in order to decrease the multi-hop packet delays caused by the longer wake-up duty cycles. Latency optimization for non-PSM stations was addressed in [21], where waves of RTS/CTS frames are proposed to reserve radio resources along the route for latency-optimized multihop communication.

\section{CONCLUSIONS}

The Future Internet of Things will connect not only Zigbeeenabled devices, such as sensors, but also consumer electronics that predominantly uses Wi-Fi for network connectivity. The power saving mechanisms of the IEEE 802.11 MAC have to be 
further optimized to enable low-cost battery-powered devices, such as electronic toys, to connect to each other directly without infrastructure support. In this paper, we proposed $\mathrm{MH}-$ PSM, an extension of the standard IEEE 802.11 PSM that enables low-latency communication over multiple hops. At the same time, MH-PSM increases the doze time ratio and, therefore, extends the battery lifetime of the devices. Using simulations, we showed the effectiveness of the proposed scheme. MH-PSM is software implementable since it does not require changes to the lower MAC. It is also backwardcompatible with the standard PSM, which guarantees interoperability with legacy devices.

\section{ACKNOWLEDGEMENT}

This work was partially funded by the European Union Seventh Framework Programme (FP7-ICT/2007-2013) under grant agreement number 288879 (Calipso; see http://www.ictcalipso.eu/).

\section{REFERENCES}

[1] Part 11: Wireless LAN Medium Access Control (MAC) and Physical Layer (PHY) Specifications, IEEE Std., Rev. IEEE Std 802.11-2012, 2012.

[2] D.-Y. Kim and C.-H. Choi, "Adaptive power management for IEEE 802.11-based ad hoc networks," in Proc. 5th World Wireless Congress, San Francisco, USA, May 2004.

[3] E.-S. Jung and N. H. Vaidya, "Improving IEEE 802.11 power saving mechanism," Wireless Networks, vol. 14, no. 3, pp. 375-391, 2008.

[4] S. Yongsheng and T. A. Gulliver, "An energy-efficient MAC protocol for ad hoc networks," Wireless Sensor Network, vol. 1, no. 5, pp. 407-416, 2009.

[5] H. Woesner, J.-P. Ebert, M. Schlager, and A. Wolisz, "Power-saving mechanisms in emerging standards for wireless LANs: the MAC level perspective," IEEE Personal Comm., vol. 5, no. 3, pp. 40-48, 1998.

[6] T. Nartem, E. Nordmark, W. Simpson, and H. Soliman, "Neighbor discovery for IP version 6 (IPv6)," RFC 4861, Sept. 2007.

[7] M. Grajzer, "Nd++ - an extended IPv6 Neighbor Discovery protocol for duplicate address detection to support stateless address autoconfiguration in IPv6 mobile ad-hoc networks," Internet-Draft, March 2011.

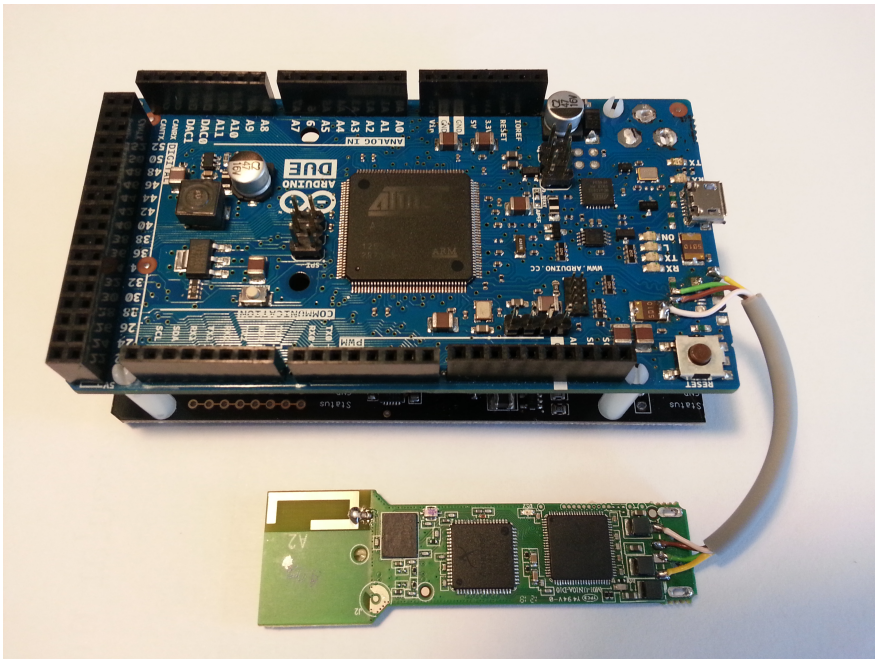

Fig. 13. Hardware platform for MH-PSM evaluation consists of an Arduino Due board and an Atheros AR9170-based Wi-Fi transceiver.

[8] Z. Shelby, S. Chaktabarti, and E. Nordmark, "Neighbor discovery optimization for Low Power and Lossy Networks (6LoWPAN)," InternetDraft, Aug. 2012.

[9] S. Mangold, "Jemula802," https://github.com/schmist/Jemula802. [Apr$2013]$.

[10] "Arduino Due," http://arduino.cc/en/Main/ArduinoBoardDue. [Apr2013].

[11] "Atheros AR9001U," http://wikidevi.com/files/Atheros/specsheets/ AR9001U.pdf. [Apr-2013].

[12] “Contiki OS," http://www.contiki-os.org/. [Apr-2013].

[13] S. Aust, R. Prasad, and I. G. M. M. Niemegeers, "IEEE 802.11ah: Advantages in standards and further challenges for sub $1 \mathrm{GHz} \mathrm{Wi}-\mathrm{Fi}$," in Proc. IEEE Int. Conf. Comm. (ICC), Ottawa, Canada, 2012.

[14] A. K. Sharma, A. Gupta, and A. Misra, "Optimized power saving mechanism for wireless ad hoc networks," in Proc. 1st Int. Conf. Recent Advances in Inf. Tech. (RAIT), Dhanbad, India, Mar. 2012.

[15] E.-S. Jung and N. Vaidya, "An energy efficient MAC protocol for wireless LANs," in Proc. IEEE Infocom, New York, USA, 2002.

[16] N. Rajangopalan and C. Mala, "Modified power save model for better energy efficiency and reduced packet latency," American Journal of Engineering and Applied Sciences, vol. 5, no. 3, pp. 237-242, 2012.

[17] J.-M. Choi, Y.-B. Ko, and J.-H. Kim, "Enhanced power saving scheme for IEEE 802.11 DCF based wireless networks," in Personal Wireless Comm., ser. Lecture Notes in Computer Science, 2003, vol. 2775, pp. 835-840.

[18] W. Akkari, A. Belghith, and A. Ben Mnaouer, "Enhancing power saving mechanisms for ad hoc networks using neighborhood information," in Proc. Int. Wireless Comm. and Mobile Comp. Conf. (IWCMC), Crete, Greece, Aug. 2008.

[19] A. Belghith and W. Akkari, "Neighborhood aware power saving mechanisms for ad hoc networks," in Proc. IEEE Conf. Local Computer Networks (LCN), Montreal, Canada, Oct. 2008.

[20] R.-H. Hwang, C.-Y. Wang, C.-J. Wu, and G.-N. Chen, "A novel efficient power-saving MAC protocol for multi-hop MANETs," Int. Journal of Comm. Systems, vol. 26, no. 1, pp. 34-55, Jan. 2013.

[21] G. Hiertz, J. Habetha, E. Weiss, and S. Mangold, "A cut-through switching technology for IEEE 802.11," in Proc. IEEE Circuits and Systems Symp. on Emerging Technologies, Shanghai, China, 2004. 